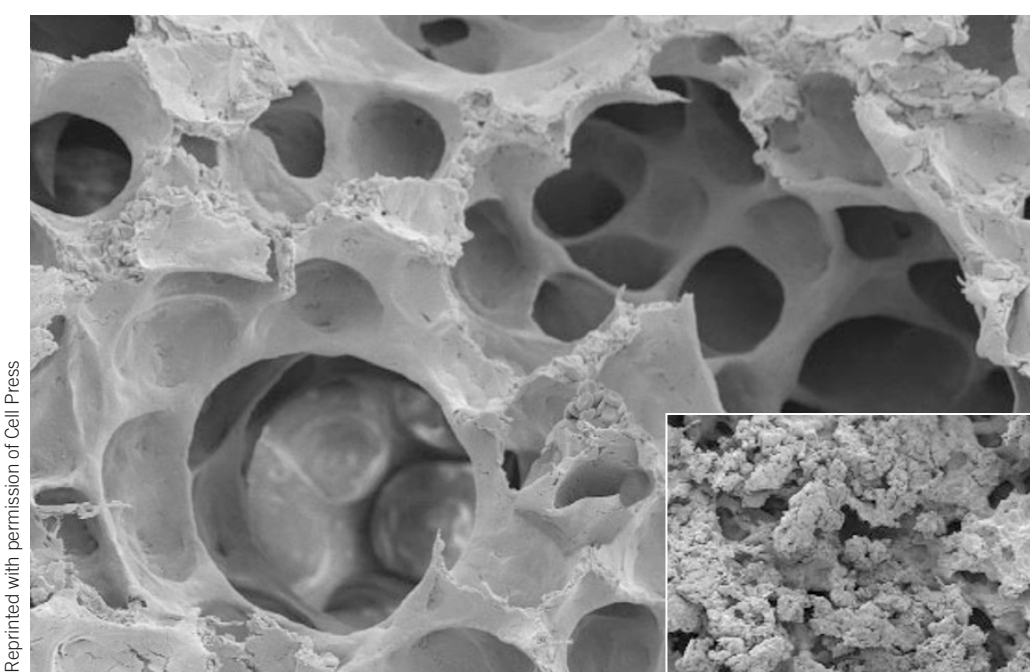

Figure 2 Scanning electron micrograph of normal lung tissue. Insert, tissue from mice exposed to $\mathrm{M}$ protein.

receptors. The result is activation of the T cells and massive release of proinflammatory cytokines $^{4}$ (Fig. 1). The results of Herwald et al. ${ }^{1}$ direct attention to the cell-surface $\mathrm{M}$ protein. This has long been recognized as an important somatic virulence factor of streptococci: strains rich in $M$ protein are antiphagocytic and are associated with more severe disease $^{5}$. But it now appears that $\mathrm{M}$ protein also has a role in causing vascular permeability.

There are more than 90 antigenic variants of $\mathrm{M}$ protein, but patients with STSS are most often infected with strains expressing the M1 protein $^{3}$, the strain used by Herwald et al. in their experiments. Previous work from the Björck laboratory had shown that a bacterial cysteine protease cleaves the M1 protein from the bacterial surface ${ }^{6}$, and that free $\mathrm{M} 1$ protein can bind fibrinogen with high affinity ${ }^{7}$. The new key finding was that complexes formed by M1 and fibrinogen bind to $\beta_{2}$-integrins on the surface of neutrophils, causing them to be activated and release heparin-binding protein. When mice were injected with purified M1 protein, or infected with an M1 strain of $S$. pyogenes, they developed severely inflamed and damaged lungs (Fig. 2). But if the mice were treated with peptide antagonists of $\beta_{2^{-}}$ integrins, they were protected.

Björck and colleagues also examined a patient who had necrotizing fasciitis caused by $S$. pyogenes. They found complexes of M1 protein and fibrinogen in tissues where they could interact with neutrophils, suggesting that their in vitro findings could be of clinical importance.

But there are many more people that are colonized by, or have minor infections caused by, M1-positive S. pyogenes than ever get STSS or necrotizing fasciitis. Why should this be? Studies of patients with severe complications of streptococcal infection showed that they are enriched for certain human leukocyte antigen haplotypes ${ }^{8}$. It has recently been shown that the streptococcal superantigenic toxins bind preferentially to certain MHC class II molecules, and that subsequent cross-linking to $\mathrm{T}$ cells is reflected in more potent T-cell activation and a greater inflammatory response ${ }^{9}$. Thus, these two recent lines of work have taken us much further forward in understand-

\author{
Liisa K Selin \& Markus Cornberg
}

The body remembers its insults. After a viral infection, memory $\mathrm{T}$ cells scatter throughout the body, ready to launch an immediate attack against reinfection in the gut, lungs, skin and other sites. These peripheral $\mathrm{T}$ cells stay at their posts for

Liisa K. Selin and Markus Cornberg are in the Department of Pathology, University of

Massachusetts Medical School, Worcester,

Massachusetts 01655, USA.

e-mail: Liisa.Selin@umassmed.edu ing the complex pathogenesis of this disease. But what of the clinical implications?

What these studies demonstrate most clearly is that 'septic shock' is not best thought of as a single disease, with a single pathogenetic mechanism that is readily susceptible to a single therapeutic intervention. For instance, the present study showed that endotoxin-the toxic component of Gram-negative bacteria such as Escherichia coli, which also cause septic shock-does not cause the release of heparin-binding protein from neutrophils. Indeed, previous attempts to treat Gram-negative septic shock with inhibitors of $\beta_{2}$-integrins have failed ${ }^{10}$.

The challenge to clinicians will be to develop diagnostic tools that will be quick and accurate enough to distinguish between the different types of septic shock, so that the most appropriate type of treatment can be used.

1. Herwald, H. et al. Cell 116, 367-379 (2004)

2. Bisno, A.L. \& Stevens, D.L. N. Engl. J. Med. 334, 240-245 (1996).

3. O'Brien, K.L. et al. Clin. Infect. Dis. 35, 268-276 (2002).

4. Lavoie, P.M., Thibodeau, J., Erard, F. \& Sekaly, R.P. Immunol. Rev. 168, 257-269 (1999).

5. Cunningham, M.W. Clin. Microbiol. Rev. 13 470-511 (2000)

6. Berge, A. \& Björck, L. J. Biol. Chem. 270, 9862-9867 (1995).

7. Åkesson, P., Schmidt, K.H., Cooney, J. \& Björck, L. Biochem. J. 300, 877-886 (1994).

8. Kotb, M. et al. Nat. Med. 8, 1398-1404 (2002).

9. Llewelyn, M. et al. J. Immunol. 172, 1719-1726 (2004).

10. Eichacker, P.Q. et al. J. Appl. Physiol. 74, 1885-1892 (1993).

\title{
Embedding $T$ cells in the matrix
}

\section{Memory T cells in peripheral tissues such as the lung lie in wait, ready to attack pathogens that try to reinvade the body. Work on mice infected with the influenza virus now identifies a molecule that keeps these $T$ cells in their positions, and prevents them from dying off.}

months; sometimes years. We now have a better idea of what keeps them there.

In the February issue of Immunity, Ray et al. ${ }^{1}$ show that the adhesion molecule very late antigen-1 (VLA-1; also called $\alpha_{1} \beta_{1}$-integrin) enables the retention and survival of $\mathrm{CD} 8^{+} \mathrm{T}$ cells directed against influenza $\mathrm{A}$ in the lung. Their results have implications for designing vaccines against this and other hard-to-beat viruses. The findings should also spur thinking on how to hinder peripheral $\mathrm{T}$ cells in diseases where memory $\mathrm{T}$ cells are out of control, such as arthritis or diabetes. 
There are two sides to T-cells. Acute Tcell responses during viral infection help clear the virus, but they also mediate lethal pathology, such as pneumonia in the case of influenza A or meningitis in the case of lymphocytic choriomeningitis virus $(\mathrm{LCMV})^{2-4}$.

Rapidly mobilized memory T-cell responses can be particularly important in mediating protective immunity upon reexposure to heterosubtypic influenza virus strains-strains with different coat proteins that evade neutralizing antibody responses. Humoral immune responses target the viral surface proteins neuraminidase and hemagglutinin, sites of frequent mutations that are not cross-protective and that define the new heterosubtypic strains of influenza that develop each year. Memory $\mathrm{CD} 8^{+} \mathrm{T}$-cell responses, on the other hand, target the more conserved internal viral epitopes-matrix and nucleoprotein-and thus can protect against heterologous strains.

After infection, $\mathrm{T}$ cells disperse throughout the host not only into lymphoid organs but also, at an even greater frequency, into nonlymphoid organs ${ }^{5-8}$. Most memory $\mathrm{T}$ cells in peripheral sites, unlike $\mathrm{T}$ cells in lymphoid organs, retain the characteristics of effector lymphocytes, such as immediate cytotoxic function and cytokine production, suggesting that they are instantly active against an invading pathogen. Thus, peripheral memory $\mathrm{T}$ cells are an ideal early first line of defense against an invading pathogen. In fact, studies have shown that immunity to a heterosubtypic strain of influenza A, which has no cross-neutralizing antibodies but identical T-cell epitopes, disappears about six months after influenza $\mathrm{A}$ infection, after antigen-specific memory $\mathrm{T}$ cells in the lung have declined ${ }^{9}$. Other studies on the LCMV infection model have shown that after the decline of peripheral effector-type memory $\mathrm{T}$ cells functional memory is also compromised to a peripheral challenge, despite intact central lymphoid memory ${ }^{10}$.

Very little is known about the factors that retain these activated memory $\mathrm{T}$ cells in the peripheral compartments. Peripheral memory $\mathrm{T}$ cells seem to be more resistant to apoptosis than lymphoid memory cells ${ }^{11}$, and they do not readily leave peripheral sites such as the lung and return to the circulation ${ }^{12}$.

Earlier work on VLA-1 showed that it may be intricately involved in T-cell immunity ${ }^{13,14}$. For instance, viral infections can increase the expression of VLA-1 and other adhesion molecules on many leukocyte

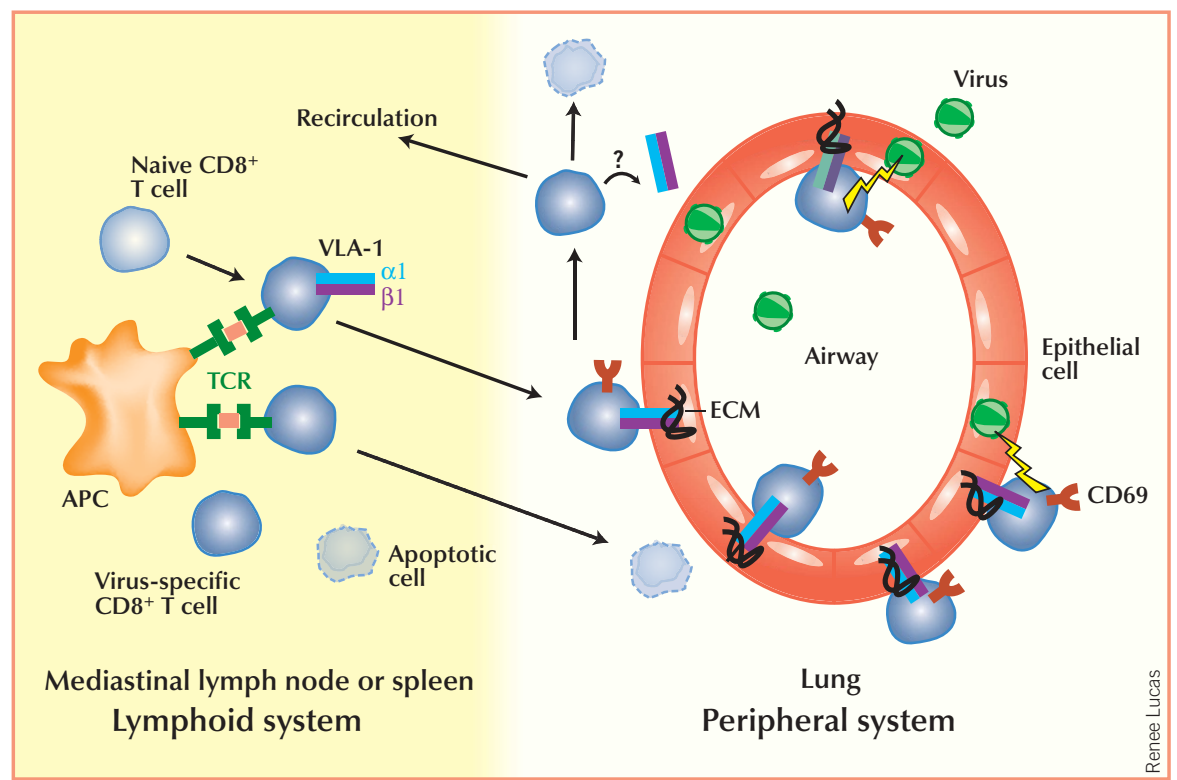

Figure 1 The role of VLA-1 in retention and survival of virus-specific memory CD8 ${ }^{+} \mathrm{T}$ cells in the lung. After activation of naive, virus-specific $\mathrm{CD}^{+} \mathrm{T}$ cells in the lymphoid compartment, Ray et al. show that VLA-1 expression is enhanced on T cells. These VLA-1 $1^{+}$T cells migrate into peripheral sites such as the lung and bind to their ligands, collagens I and IV, in the extracellular matrix (ECM) on epithelial cells of the lung airways. This interaction results in retention and increased survival of virusspecific memory T cells in the lung. These cells are then readily available as a first line of defense against reinfection with the virus. APC, antigen-presenting cell; TCR, T-cell receptor.

populations, and can influence T-cell activation. The ligands for VLA-1 are collagens I and IV, which populate the extracellular matrix (ECM) of all peripheral organs. For example, collagen fibrils make up $15 \%$ of the lung by dry weight. Thus, T cells migrating through peripheral tissues are constantly in contact with collagen. Histological examination shows that lymphocytes localize near epithelial or endothelial boundaries near the basement membrane, which is rich in type IV collagen.

Interestingly, the ECM is separated from the T-cell compartments by the fibroblastic reticular network, so cells in the spleen and lymph nodes do not come in contact with the ECM. This could affect their interactions with antigen-presenting cells. In the presence of collagen, as in peripheral tissues, $\mathrm{T}$ cells form only transient interactions with antigen-presenting cells. This would favor the activation of memory $\mathrm{T}$ cells over naive $\mathrm{T}$ cells, which require prolonged periods of contact. Thus, it is possible that naive cells are best activated in the lymphoid compartment, because in the absence of collagen contact they are free to interact with dendritic cells for the prolonged periods required for T-cell activation.

In vitro studies have shown that VLA-1 modulates T-cell activation, as VLA-1 interaction with collagens I and IV enhances cytokine production and proliferation triggered through the T-cell receptor. Antibody blocking studies implicate VLA-1 in several in vivo models of inflammation mediated by $\mathrm{T}$ cells, including LCMV-induced delayed-type hypersensitivity responses and collagen-induced arthritis ${ }^{13,14}$.

Ray et al. showed that during acute influenza A infection, virus-specific $\mathrm{CD}^{+}$ $\mathrm{T}$ cells increased expression of VLA-1 and were preferentially retained in peripheral organs, including regions of the lung airways and tissue (bronchioalveolar lymphocytes and lung parenchyma; Fig. 1). VLA-1 expression on $\mathrm{T}$ cells, however, was not required for the initial trafficking into peripheral tissues. Histological staining revealed that $\mathrm{VLA}-{ }^{+} \mathrm{CD} 8{ }^{+} \mathrm{T}$ cells congregated at sites of high ECM content near bronchioles and vessels, suggesting that these cells were interacting with their ligands, collagens I and IV.

These $\mathrm{T}$ cells in contact with ECM through VLA-1 seemed to have features of an effector phenotype, expressing high CD69 and low CD62L. They were also less prone to apoptosis than VLA- $1^{-}$antigenspecific cells, as shown by TUNEL staining. These results suggest that the interaction of VLA- 1 on memory $T$ cells with its ligand in the ECM enhances both survival and retention of these cells in the lung. 
The authors went on to show that VLA-1 is crucial for protective immunity to heterologous influenza virus infection. That was made clear by using a blocking antibody to VLA- 1 and by analyzing mice genetically lacking VLA-1. Interestingly, although VLA1 knockout mice had more influenza A-specific $\mathrm{CD}^{+}$memory $\mathrm{T}$ cells in the spleen than normal mice, they had no protective immunity.

What is not yet clear is exactly how VLA-1 enhances cell survival and maintains the effector phenotype. Does VLA-1 initiate an intracellular signaling process, or does attachment to collagen somehow protect these cells from other, potentially lethal, cellular interactions? The mechanism behind the decline of memory $\mathrm{T}$ cells over time also remains to be identified. Memory $\mathrm{T}$ cells may ultimately lose VLA-1 expression and return to the circulation, or the VLA-1/collagen interaction may provide only limited protection from apoptosis.

This study could enhance efforts to develop immunotherapy against T-cellmediated diseases and create new vaccines. Treatments that interfere with the interac- tion of T-cell VLA-1 with collagen may diminish the immunopathological symptoms of diseases like asthma and rheumatoid arthritis.

Using the newly developed, intranasally administered, live attenuated influenza vaccine that also stimulates cellular immune responses directly in the mucosa of the respiratory tract could have added benefits over more conventional vaccines. The conventional killed influenza vaccine is administered intramuscularly and relies on the induction of humoral immune responses; the vaccine does not provide protection against new heterosubtypic influenza strains.

The live attenuated vaccine, only recently introduced into clinics, could make the difference between life and death for some individuals. A live vaccine has the benefit of more conserved, cross-protective $\mathrm{CD}^{+} \mathrm{T}$ cell responses that could provide at least partial protection against new emerging strains. The new avian influenza is one such strain; it has the potential to cause the next influenza pandemic, and is the focus of intensive vaccine development efforts.
A note of caution is in order: repeated doses of a vaccine that maintains high numbers of memory T cells in the lung could also amplify immunopathology after repeated infection by heterologous viruses ${ }^{15}$.

1. Ray, S.J. et al. Immunity 20, 167-179 (2004).

2. Effros, R.B., Doherty, P.C., Gerhard, W. \& Bennick, J.R. J. Exp. Med. 145, 557-568 (1977).

3. Klavinskas, L.S., Whitton, L.J., Joly, E. \& Oldstone, M.B.A. Virology 178, 393-400 (1990).

4. Doherty, P.C. \& Zinkernagel, R.M. Transplant. Rev. 19, 89-120 (1974).

5. Marshall, D.R. et al. Proc. Natl. Acad. Sci. USA 98, 6313-6318 (2001).

6. Masopust, D., Vezys, V., Marzo,A.L. \& Lefrancois, L. Science 291, 2413-2417 (2001).

7. Hogan, R.J. et al. J. Immunol. 166, 1813-1822 (2001).

8. Chen, H.D. et al. Nat. Immunol. 2, 1067-1076 (2001).

9. Liang, S., Mozdzanowska, K., Palladino,G. \& Gerhard, W. J. Immunol. 152, 1653-1661 (1994).

10. Bachmann, M.F., Kundig, T.M., Hengartner, H. \& Zinkernagel, R.M. Proc. Natl. Acad. Sci. USA 94, 640-645 (1997).

11. Wang, X.Z. et al. Immunity 18, 631-642 (2003).

12. Harris, N.L., Watt, V., Ronchese, F. \& Le Gros, G. J. Exp. Med. 195, 317-326 (2002).

13. Andreasan, S.O. et al. J. Immunol. 171 2804-2811 (2003).

14. Dustin, M.L. \& De Fougerolles, A.R. Curr. Opin. Immunol. 13, 286-290 (2001).

15. Welsh, R.M. \& Selin, L.K. Nat. Rev. Immunol. 2, 417-426 (2002).

\title{
Putting the heat on ALS
}

\author{
Susanna C Benn \& Robert H Brown, Jr
}

\section{An enhancer of the heat shock response alleviates symptoms of neurodegeneration and prolongs lifespan in a mouse model of amyotrophic lateral sclerosis-even when administered after onset (pages 402-405).}

In patients with amyotrophic lateral sclerosis (ALS), also known as Lou Gehrig disease, degeneration of motor neurons causes progressive muscular weakness, atrophy and paralysis. Respiratory failure inexorably leads to death, typically within four or five years. There is no definitive treatment for this disease; riluzole, the only drug approved for ALS, is at best palliative and prolongs survival by only $10-15 \%$. The cause of most cases of ALS is not defined, but a small subset of cases-about 2-3\%-are caused by missense mutations in the gene encoding cytosolic $\mathrm{Cu} / \mathrm{Zn}$ superoxide dismutase-1 (SOD-1).

Susanna Benn and Robert H. Brown, Jr. are in the Day Laboratory for Neuromuscular Research, Massachusetts General Hospital, Charlestown, Massachusetts 02129, USA. e-mail: sbenn@partners.org or rhbrown@partners.org
In this issue, Kieran et al. ${ }^{1}$ provide evidence that a new category of molecules may ameliorate the disease. These investigators show that daily treatments with arimoclomol, a coinducer of heat shock proteins, slows the process of motor neuron death and thereby extends survival in a transgenic SOD $1^{\mathrm{G} 93 \mathrm{~A}}$ mouse model of ALS (Fig. 1).

Several indices of motor neuron viability, including cell counts and measurements of motor unit number and muscle fatigue index (a measure of early motor neuron dysfunction), confirm the benefit of arimoclomol, particularly for large motor neurons. The treatment is remarkable both for its degree of effectiveness and because it works on mice that are already symptomatic.

Despite intensive investigations, the salient pathogenic mechanisms have not been identified with certainty for any type of ALS. It is clear in transgenic rodents that high levels of mutant SOD-1 protein kill motor neurons in a dose-dependent manner. In these models, multiple processes contribute to the demise of motor neurons, including early misfolding and aggregation of mutant SOD-1 protein ${ }^{2}$, excitotoxicity mediated by failing synaptic transport of glutamate into glial cells, diminished energy generation due to mitochondrial dysfunction, and impaired axonal transport.

In both rodents and humans, afflicted motor neurons prompt proliferation of surrounding astrocytes and microglial cells in affected spinal gray matter. Gene expression and biochemical studies reveal hallmarks of inflammation, such as activation of cyclooxygenase. These findings are evident in both sporadic ALS cases and those associated with mutant SOD-1. The motor neuron death process is not cell-autonomous. Rather, it is intimately related to the context of surrounding cells; cell death of motor 